\begin{tabular}{|c|l|}
\hline Title & Efficient optical coupling into a single plasmonic nanostructure using a fiber-coupled microspherical cavity \\
\hline Author(s) & Takashima, Hideaki; Kitajima, Kazutaka; Tanaka, Yoshito; Fujiwara, Hideki; Sasaki, Keiji \\
\hline Citation & $\begin{array}{l}\text { Physical Review A, 89(2), 021801-1-021801-5 } \\
\text { https://doi.org/10.1103/PhysRevA.89.021801 }\end{array}$ \\
\hline Issue Date & 2014.02-05 \\
\hline Doc URL & http://hdl.handle.net/2115/63205 \\
\hline Rights & ○ 2014 A merican Physical Society \\
\hline Type & article \\
\hline File Information & PhysRevA.89.021801.pdf \\
\hline
\end{tabular}

Instructions for use 


\title{
Efficient optical coupling into a single plasmonic nanostructure using a fiber-coupled microspherical cavity
}

\author{
Hideaki Takashima, Kazutaka Kitajima, Yoshito Tanaka, Hideki Fujiwara, and Keiji Sasaki* \\ Research Institute for Electronic Science, Hokkaido University, Kita-20, Nishi-10, Kita-ku, Sapporo, Hokkaido 001-0020 Japan
}

(Received 21 August 2012; revised manuscript received 20 September 2013; published 5 February 2014)

\begin{abstract}
Toward complete coupling between propagating light (PL) and a single localized-surface-plasmon (LSP) nanostructure, we propose a tapered-fiber-coupled microspherical cavity system combining an Au-coated probe tip. This system possesses the unique characteristic of precise adjustability for the fiber-cavity coupling rate and the cavity-plasmon coupling rate, which is indispensable for achieving the critical coupling conditions. We successfully demonstrate the $93 \%$ PL coupling into the LSP antenna with an effective area of a $58 \mathrm{~nm}$ circle, exceeding the diffraction limit.
\end{abstract}

DOI: 10.1103/PhysRevA.89.021801

PACS number(s): 42.81.Qb, 73.20.Mf, 42.60.Da

Localized surface plasmons (LSPs) of metal nanostructures have attracted considerable attention because of the unique enhancement of the exciton-photon coupling by strong localization of the optical field [1-11]. The LSP polaritons have the ability to confine the optical field into nanometer-scale areas, exceeding the diffraction limit, using the so-called optical antenna effect [1-3]. LSP fields with the small mode volumes strongly enhance the interactions between light and matter [4-8]. Recently, vacuum Rabi splitting was observed in exciton-plasmon coupling systems at room temperature [9-11]. This effect demonstrates that a single photon harvested by the LSP antenna can couple into a single-exciton state with high efficiency, which will be applicable to highly efficient photonic devices. One of the ultimate goals is a single-photon switching gate for quantum information $[12,13]$.

Although the strong-coupling regime can be obtained within the LSP field, the coupling efficiency between a single LSP antenna and propagating light (PL) in free space or in optical waveguides is extremely low. The harvesting antenna size, which corresponds to the extinction cross section of a single metal nanostructure, is typically $\sim 10^{3} \mathrm{~nm}^{2}$ for visible light [14], which is comparable to the actual geometrical cross section of the structure. The desired size of a single antenna structure is on the order of tens of nanometers, which is determined from the LSP resonance wavelength. Conversely, the cross section of the PL mode is $\sim 10^{5} \mathrm{~nm}^{2}$ for a diffraction-limited spot and $\sim 10^{7} \mathrm{~nm}^{2}$ for a single-mode optical fiber. This size mismatch between the LSP antenna and the PL mode results in a low coupling efficiency on the order of $\sim 10^{-4}-10^{-2}$, which limits the total throughput of the exciton-photon coupling via the LSP polariton.

The use of microcavities can drastically improve the efficiency of the optical coupling with metal nanostructures. There are some reports on the cavity-LSP coupling systems and their applications to highly sensitive biosensors and efficient photon emitters [15-19]. Those works, however, have not paid attention to ultimate optimization of the coupling between the PL mode and the LSP antenna via the microcavity mode [20,21]. Toward complete PL-LSP coupling, which means that all power of PL can couple into a single LSP antenna without loss, we employ a tapered-fiber-coupled microspherical cavity. Microspherical cavities possess ultra-high-quality factors $\left(>10^{8}\right)$ and small mode volumes $\left(<10^{3} \mu \mathrm{m}^{3}\right)$ [22-25], which can provide sufficiently high intracavity enhancement for strong PL-LSP coupling. Because the whispering gallery mode forms an evanescent field around the microsphere, a metal nanostructure placed on or near the sphere surface can be coupled with the cavity mode. By changing the separation distance between the sphere and the metal structure, the coupling constant between the cavity mode and the LSP polariton can be adjusted precisely. And, the optical tunneling between the tapered fiber and the sphere can be controlled with tuning the distance. This adjustability is indispensable for attaining the critical (complete) coupling condition. Here, we experimentally demonstrate the applicability for the highly efficient PL coupling into an LSP antenna with a minute cross section.

In this study, the PL-LSP coupling efficiency $G$ is defined as the ratio between the power of light coupled into a single metal nanostructure $P_{2}$ and the power of incident light on the tapered fiber $P_{1}$. The efficiency $G$ at the cavity resonance is theoretically given by

$$
\begin{aligned}
G & =\frac{P_{2}}{P_{1}}=\frac{4 \gamma_{1} \gamma_{2}}{\left(\gamma_{1}+\gamma_{2}+\gamma_{c}\right)^{2}} \\
& =\left\{1+\frac{\left(\sqrt{\gamma_{1}}-\sqrt{\gamma_{2}}\right)^{2}+\gamma_{c}}{2 \sqrt{\gamma_{1} \gamma_{2}}}\right\}^{-2},
\end{aligned}
$$

where $\gamma_{1}$ represents the coupling rate between the PL mode of the tapered fiber and the resonance mode of the spherical cavity, $\gamma_{2}$ is the coupling rate from the cavity mode to the LSP antenna, and $\gamma_{c}$ is the rate of the intrinsic cavity loss including the coupling loss to the higher-order fiber modes (see Fig. 1). These coupling rates are assumed to be much smaller than the inverse of the cavity round-trip time, i.e., $\gamma_{1}, \gamma_{2}, \gamma_{\mathrm{c}} \ll c / l_{c}$, where $l_{c}$ denotes the effective cavity length and $c$ is the speed of light. This approximation is valid for the experimental system described below. Equation (1) is derived from the resonance condition on the cavity field as

$$
\sqrt{P_{c}}=\sqrt{\left\{1-\frac{l_{c}}{c}\left(\gamma_{1}+\gamma_{2}+\gamma_{c}\right)\right\} P_{c}}+\sqrt{\frac{l_{c}}{c} \gamma_{1} P_{1}},
$$






FIG. 1. (Color online) Optical coupling into a metal nanostructure using a tapered-fiber-coupled microspherical cavity. The doubleheaded arrows show the direction of the electric field (TM mode). Propagating light in a tapered fiber is coupled to a microspherical cavity, and a single metal nanostructure (LSP antenna) placed on or near the microsphere surface can be coupled to the cavity mode. By adjusting the coupling parameters, the critical PL-LSP coupling is realized such that the whole power of incident light can be harvested by the LSP antenna.

and the output coupling condition as

$$
\sqrt{P_{2}}=\sqrt{\frac{l_{c}}{c} \gamma_{2} P_{c}},
$$

where $P_{\mathrm{c}}$ is the power of light confined in the cavity. The first and the last terms in Eq. (2) represent the output from the cavity and input from the tapered fiber, respectively [26]. The cavity-LSP coupling rate $\gamma_{2}$ can be determined from

$$
\gamma_{2}=\frac{c}{l_{c}} \frac{\sigma}{S}
$$

where $S$ is the cross section of the whispering gallery mode, and $\sigma$ is the effective extinction cross section of the LSP antenna, which is a function of the antenna position within the cavity mode. We note that the extinction cross section consists of scattering and absorption components, and both of these loss processes are intermediated by the LSP state.

According to Eq. (1), the required conditions for realizing the critical PL-LSP coupling, in which the coupling efficiency is maximized $(G=1)$, are

$$
\gamma_{1}=\gamma_{2} \gg \gamma_{c}
$$

The coupling rates can be experimentally estimated from the linewidths of resonant dips in transmission spectra measured at the output port of the tapered fiber. The relation between the linewidth $\Delta v$ and the coupling rates is expressed as

$$
\Delta v=\frac{1}{2 \pi}\left(\gamma_{1}+\gamma_{2}+\gamma_{c}\right) .
$$

The transmittance $T$ of the fiber output power at the resonance wavelength is also a function of the coupling rates,

$$
T=\left(\frac{\gamma_{1}-\gamma_{2}-\gamma_{c}}{\gamma_{1}+\gamma_{2}+\gamma_{c}}\right)^{2} .
$$

When the intrinsic cavity loss is negligibly small with $\gamma_{1} \gg \gamma_{c}$ and $\gamma_{2} \gg \gamma_{c}$, the PL-LSP coupling efficiency is approximately given by

$$
G \approx 1-T
$$

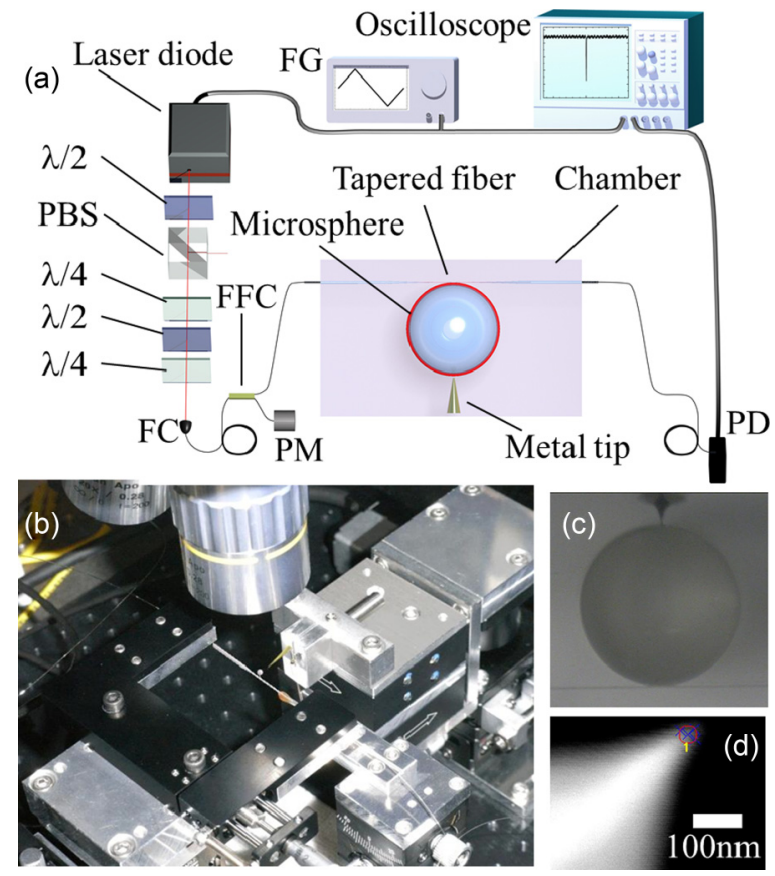

FIG. 2. (Color online) PL-LSP coupling system with a taperedfiber-coupled microspherical cavity. (a) Schematic of experimental setup. A tunable diode laser is introduced into the tapered fiber through a fiber coupler (FC), and the output intensity is detected by a photodiode (PD). The laser frequency is swept by applying a voltage from a function generator (FG) to measure the transmission spectra. The laser power is adjusted using a half-wave plate $(\lambda / 2)$ and a polarization beam splitter (PBS) and measured by an optical power meter (PM) connected to a balanced fused fiber coupler (FFC). The polarization of the incident light is adjusted using a half-wave plate $(\lambda / 2)$ and two quarter-wave plates $(\lambda / 4)$. (b) A photograph of the experimental setup around a tapered-fiber-coupled microspherical cavity with a metal tip. (c) A photograph of the microspherical cavity coupled with the tapered fiber and the metal tip. (d) A scanning electron micrograph of the Au-coated tip. The circle indicates the curvature radius of the end of the Au-coated tip, which is approximately $50 \mathrm{~nm}$.

The experimental setup and its micrographs are shown in Fig. 2. The diameter of a fused-silica microsphere is $50 \mu \mathrm{m}$. The waist diameter of a tapered fiber is $\sim 400 \mathrm{~nm}$, which maintains single-mode propagation in the tapered fiber. For the LSP antenna, we employ an atomic force microscope (AFM) probe tip with metal coating. The tip is prepared by depositing an $\mathrm{Au}$ film on a commercial Si AFM probe. The film thickness is designed to be $50 \mathrm{~nm}$ [see Fig. 2(d)]. The scattering spectrum of this probe exhibits a resonant peak at $730 \mathrm{~nm}$, and the full width at half maximum of the peak is $\sim 200 \mathrm{~nm} \mathrm{[27].} \mathrm{The}$ tapered fiber and the Au-coated tip are positioned at opposite sides of the equator of the microsphere [Figs. 2(b) and 2(c)], and their separation distances from the sphere surface are individually controlled using the feedback-controlled threedimensional piezo manipulators with the nanometer precisions [28]. The zero distance is determined as the position where the spectral change is stopped in the fiber- and tip-approaching processes. This coupling cavity system is placed in an acrylic chamber that is filled with dry air (humidity $<1 \%$ ). The 


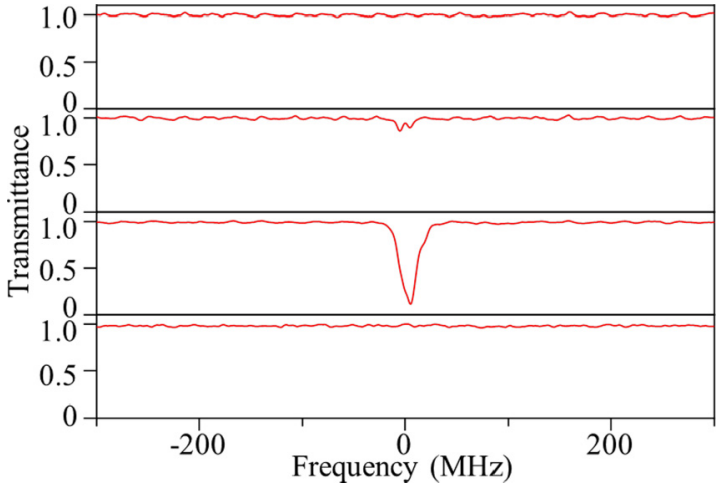

FIG. 3. (Color online) Transmission spectra of the tapered-fibercoupled microspherical cavity without the metal tip measured at four different distances. From top to bottom, the separation distances between the tapered fiber and the microsphere surface are 350, 280, 70 , and $0 \mathrm{~nm}$.

chamber is further covered with expanded polystyrene to be kept at a stable temperature $(<0.1 \mathrm{~K}$ for an hour $)$.

A tunable external-cavity diode laser with a linewidth of $300 \mathrm{kHz}$ is introduced into the input port of the tapered fiber, and the light power at the output port is detected with a photodiode. For measuring transmission spectra, the laser frequency is swept over a range of $5.1 \mathrm{GHz}$ around $780 \mathrm{~nm}$ wavelength. The laser power is less than a few $\mathrm{nW}$, so as to avoid the thermally induced shape change in resonant spectra. The polarization of the incident light is adjusted with wave plates using an experimental technique [29], such that the transverse magnetic (TM) modes can be selectively excited in the microspherical cavity.

In the first step of the experiment, we analyze the resonance property of the tapered-fiber-coupled microspherical cavity without the metal nanostructure. The transmission spectra are observed by varying the separation distance between the sphere surface and the tapered fiber, as shown in Fig. 3. When the fiber is far $(>350 \mathrm{~nm})$ from the microsphere, the cavity does not affect the spectrum and the transmittance of the tapered fiber $T_{0}$ is 0.99 . At a distance of $280 \mathrm{~nm}$, split resonant dips appear in the spectrum. This splitting can be attributed to the backscattering-aided coupling between two degenerate whispering gallery modes propagating in opposite directions [30]. The linewidth of the single dip is $2.4 \mathrm{MHz}$, which corresponds to the quality factor of $1.6 \times$ $10^{8}$. According to Eq. (6) with $\gamma_{1} \approx \gamma_{2}=0$, the intrinsic loss rate $\gamma_{\mathrm{c}}$ of the microspherical cavity is $1.51 \times 10^{7} \mathrm{~s}^{-1}$. As the separation decreases to $70 \mathrm{~nm}$, the transmittance at the resonance frequency is reduced to the minimum. At this distance, the fiber-sphere coupling rate $\gamma_{1}$ would be balanced by the cavity loss rate of $\gamma_{c}$; thus, the transmittance should be zero, but the experimental result indicates $T_{p}=0.047$. This deviation can be explained by the uncoupled light having a different polarization than the cavity mode. The polarization change in the tapered fiber cannot be eliminated completely [29]. By further decreasing the distance, the dip becomes much broader and shallower, and when the fiber is in contact with the microsphere, the spectrum is nearly flat. However, an insignificant resonant dip remains because the transmittance

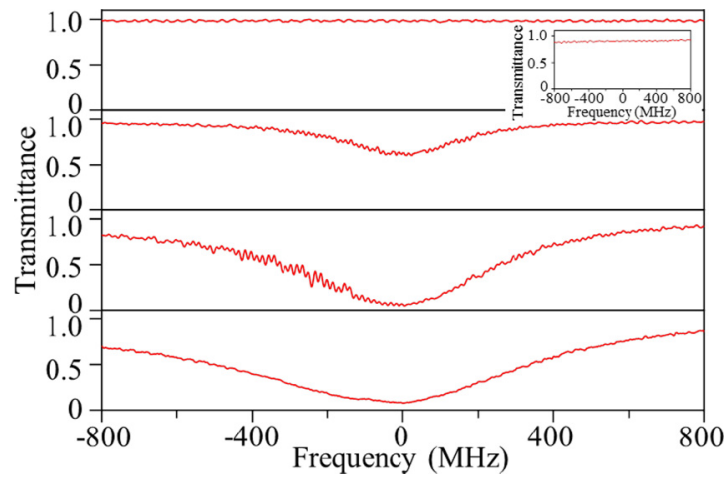

FIG. 4. (Color online) Transmission spectra of the tapered-fibercoupled microspherical cavity with the metal tip measured at four different distances. From top to bottom, the separation distances between the microsphere surface and the metal tip are 1140, 330, 50 , and $0 \mathrm{~nm}$. The inset is a transmission spectrum with uncoated AFM tip under the contact condition.

at the resonance frequency is 0.98 , which is slightly lower than $T_{0}$.

In the second step, the cavity-LSP coupling system is analyzed after including the Au-coated AFM tip as the metal nanostructure. The position of the Au-coated tip is moved toward the microsphere in $15 \mathrm{~nm}$ intervals, while contact between the tapered fiber and sphere is maintained. Figure 4 shows the transmission spectra measured at four distinct distances. At a large separation $(>1140 \mathrm{~nm})$ between the tip and the sphere surface, there is no change from the flat spectrum in the bottom of Fig. 3. When the distance is $350 \mathrm{~nm}$, the spectrum exhibits a resonant dip with a wide linewidth. The dip increases in the depth with decreasing separation. At a distance of $50 \mathrm{~nm}$, the transmittance at the resonance frequency reaches a minimum at which the critical coupling condition given by Eq. (5) is achieved, as discussed below. The minimum transmittance $T_{m}$ is 0.053 , which is almost the same as the minimum $T_{p}$ in Fig. 3 and can also be ascribed to the uncoupled polarization light. At this position, scattered light from the Au-coated tip can be clearly observed. When the Au-coated tip is even closer to the sphere, a slight decrease in the depth and a broadening of the resonant dip are observed in the spectrum. In the transmittance measurement, we simultaneously monitor the reflected light that is backward propagating in the fiber (Fig. 1), and no appreciable signal is detected. Here, we note that there is no appreciable change from the flat transmission spectrum when an AFM tip without Au coating approaches the sphere (inset of Fig. 4).

Figures 5(a) and 5(b) represent plots of the dip-bottom transmittance and the dip linewidth with respect to the metal tip-sphere distance. At the long-distance limit, the transmittance and the linewidth are asymptotically 0.98 and $280 \mathrm{MHz}$, respectively, as indicated by the green lines in Fig. 5. Substituting this linewidth and $\gamma_{2}=0$ into Eq. (6), we determine the decay rate $\left(\gamma_{1}+\gamma_{\mathrm{c}}\right)$ of the tapered-fibercontacted microspherical cavity to be $1.76 \times 10^{9} \mathrm{~s}^{-1}$, which corresponds to the quality factor of $1.4 \times 10^{6}$. Using the given $\gamma_{\mathrm{c}}$, the coupling rate $\gamma_{1}$ between the fiber PL mode and the cavity resonance mode is given as $1.74 \times 10^{9} \mathrm{~s}^{-1}$. The transmittance calculated from these coupling rates using 

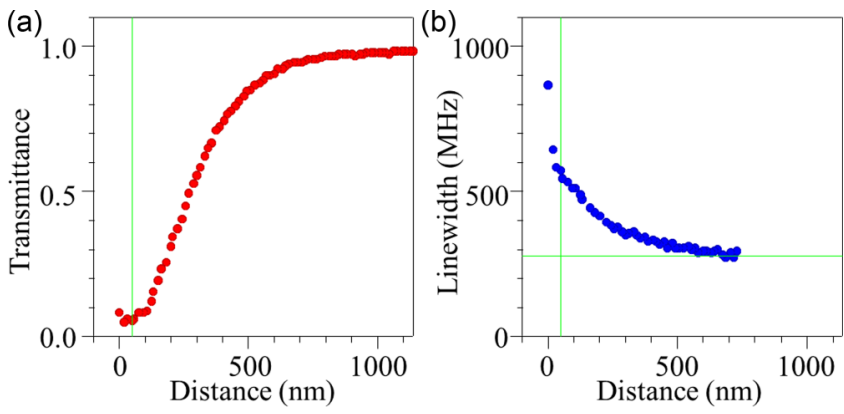

FIG. 5. (Color online) Separation distance dependence of the transmittance and linewidth. (a) The transmittance of the resonant dip as a function of the separation distance between the metal tip and the microsphere surface. (b) The resonant linewidth as a function of the separation distance. The vertical and horizontal solid green lines indicate the separation distance at the critical coupling condition and the linewidth at the long-distance limit, respectively.

Eq. (7) is 0.97, which is in good agreement with the above-mentioned experimental value, even when the fiber transmittance is considered. As the separation decreases, the transmittance is drastically reduced, and then minimized at the distance of $50 \mathrm{~nm}$, which suggests critical coupling. In contrast, the linewidth gradually increases and reaches $560 \mathrm{MHz}$ at a separation of $50 \mathrm{~nm}$. At this critical point, the total decay rate $\left(\gamma_{1}+\gamma_{2}+\gamma_{\mathrm{c}}\right)$ is $3.52 \times 10^{9} \mathrm{~s}^{-1}$, from which we obtain a coupling rate $\gamma_{2}$ between the cavity mode and the LSP polariton of $1.76 \times 10^{9} \mathrm{~s}^{-1}$. These results indicate that the critical coupling conditions of Eq. (6), $\gamma_{1}=\gamma_{2} \gg \gamma_{c}$, are successfully attained.

Using the given $\gamma_{1}, \gamma_{2}$, and $\gamma_{\mathrm{c}}$, the PL-LSP coupling efficiency $G$ is estimated from Eq. (1) as 0.99 . Given the leakage loss of the tapered fiber $(1 \%)$ and the uncoupled polarization component (5\%), the actual coupling efficiency of this experimental system is determined to be 0.93 . Thus, we conclude that a $93 \%$ efficiency can be achieved for coupling between incident light in the fiber and a single metal nanostructure. We also calculate the cross section $S$, the effective cavity length $l_{c}$, and the free spectral range of the whispering gallery mode using the finite-element method, which are $2.0 \mu \mathrm{m}^{2}, 220 \mu \mathrm{m}$, and $1.6 \mathrm{THz}$, respectively. We then determine the effective extinction cross section $\sigma$ of the LSP antenna according to Eq. (4) to be $2.6 \times 10^{3} \mathrm{~nm}^{2}$, which corresponds to a circular area with a diameter of $58 \mathrm{~nm}$. The ratio of the LSP antenna size to the cavity mode cross section is $1.3 \times 10^{-3}$, which corresponds to a $\sim 10^{3}$ enhancement of the PL-LSP coupling efficiency.

To conclude, we have successfully demonstrated nearly complete optical coupling into a single metal nanostructure using a tapered-fiber-coupled microspherical cavity system. Light propagating in an optical fiber can be confined into an LSP antenna with an effective area of a $58 \mathrm{~nm}$ circle, exceeding the diffraction limit, with an efficiency of $93 \%$. In other words, the cross section of a single plasmonic nanostructure can be effectively enlarged to a size comparable to a mode area of a waveguide, thereby bridging the three-order-of-magnitude difference in size. Finally, the well-designed combination of the microcavity enhancement and the LSP localization can realize light focusing into a single-nanometer area with perfect efficiency.

This work was supported by Grants-in-Aid for Scientific Research (Grants No. 23246016, No. 22681011, No. 21710090, No. 23656039, and No. 24651111) from the Ministry of Education, Culture, Sports, Science of Japan.
[1] H. Fischer and O. J. F. Martin, Opt. Express 16, 9144 (2008).

[2] S. Kühn, U. Håkanson, L. Rogobete, and V. Sandoghdar, Phys. Rev. Lett. 97, 017402 (2006).

[3] P. Mühlschlegel, H.-J. Eisler, O. J. F. Martin, and B. Hecht, Science 308, 1607 (2005).

[4] M. Moskovits, J. Raman Spectrosc. 36, 485 (2005).

[5] K. Kneipp, H. Kneipp, I. Itzkan, R. R. Dasari, and M. S. Feld, J. Phys.: Condens. Matter 14, R597 (2002).

[6] K. Ueno, S. Juodkazis, T. Shibuya, Y. Yokota, V. Mizeikis, K. Sasaki, and H. Misawa, J. Am. Chem. Soc. 130, 6928 (2008).

[7] T. Neumann, M.-L. Johansson, D. Kambhampati, and W. Knoll, Adv. Funct. Mater. 12, 575 (2002).

[8] F. Tam, G. P. Goodrich, B. R. Johnson, and N. J. Halas, Nano Lett. 7, 496 (2007).

[9] J. Bellessa, C. Bonnand, J. C. Plenet, and J. Mugnier, Phys. Rev. Lett. 93, 036404 (2004).

[10] S. Savasta, R. Saija, A. Ridolfo, O. Di Stefano, P. Denti, and F. Borghese, ACS Nano 4, 6369 (2010).

[11] T. K. Hakala, J. J. Toppari, A. Kuzyk, M. Pettersson, H. Tikkanen, H. Kunttu, and P. Törmä, Phys. Rev. Lett. 103, 053602 (2009).

[12] Q. A. Turchette, C. J. Hood, W. Lange, H. Mabuchi, and H. J. Kimble, Phys. Rev. Lett. 75, 4710 (1995).
[13] T. Aoki, B. Dayan, E. Wilcut, W. P. Bowen, A. S. Parkins, T. J. Kippenberg, K. J. Vahala, and H. J. Kimble, Nature (London) 443, 671 (2006).

[14] J. Yguerabide and E. E. Yguerabide, Anal. Biochem. 262, 137 (1998)

[15] S. I. Shopova, R. Rajmangal, S. Holler, and S. Arnold, Appl. Phys. Lett. 98, 243104 (2011).

[16] Dzmitry Melnikau, Diana Savateeva, Andrey Chuvilin, Rainer Hillenbrand, and Yury P. Rakovich, Opt. Express 19, 22280 (2011).

[17] Wonmi Ahn, Svetlana V. Boriskina, Yan Hong, and Björn M. Reinhard, ACS Nano 6, 951 (2012).

[18] Yun-Feng Xiao, Yong-Chun Liu, Bei-Bei Li, You-Ling Chen, Yan Li, and Qihuang Gong, Phys. Rev. A 85, 031805 (2012).

[19] F. Vollmer and L. Yang, Nanophotonics 1, 267 (2012).

[20] M. Barth, S. Schietinger, S. Fischer, J. Becker, N. Nüsse, T. Aichele, B. Löchel, C. Sönnichsen, and O. Benson, Nano Lett. 10, 891 (2010).

[21] M. Chamanzar and A. Adibi, Opt. Express 19, 22292 (2011).

[22] M. L. Gorodetsky, A. A. Savchenkov, and V. S. Ilchenko, Opt. Lett. 21, 453 (1996). 
[23] S. M. Spillane, T. J. Kippenberg, and K. J. Vahala, Nature (London) 415, 621 (2002).

[24] J. R. Buck and H. J. Kimble, Phys. Rev. A 67, 033806 (2003).

[25] A. Chiba, H. Fujiwara, J. Hotta, S. Takeuchi, and K. Sasaki, Appl. Phys. Lett. 86, 261106 (2005).

[26] S. M. Spillane, T. J. Kippenberg, O. J. Painter, and K. J. Vahala, Phys. Rev. Lett. 91, 043902 (2003).

[27] F. Ren, H. Takashima, Y. Tanaka, H. Fujiwara, and K. Sasaki, Opt. Express 21, 27759 (2013).
[28] We determined their separation distances from the position information of the piezo manipulators since the difference between the position information and the actual distance was negligibly small for the suppression of the thermal fluctuation and the mechanical vibration of the system.

[29] H. Konishi, H. Fujiwara, S. Takeuchi, and K. Sasaki, Appl. Phys. Lett. 89, 121107 (2006).

[30] J. Zhu, S. K. Ozdemir, Y. F. Xiao, L. Li, L. He, D. R. Chen, and L. Yang, Nat. Photon. 4, 46 (2010). 